\title{
The age-activity-rotation relationship in solar-type stars ${ }^{\star}$
}

\author{
G. Pace ${ }^{1,2}$ and L. Pasquini ${ }^{1}$ \\ 1 European Southern Observatory, Karl Schwarzschild Strasse 2, 85748, Garching bei München, Germany \\ e-mail: gpace@eso.org \\ 2 Dipartimento di Astronomia, Università di Trieste, via GB Tiepolo 11, 34131 Trieste, Italy
}

Received 31 March 2004 / Accepted 27 June 2004

\begin{abstract}
We present Ca II K line chromospheric fluxes measured from high-resolution spectra in $35 \mathrm{G}$ dwarf stars of 5 open clusters to determine the age-activity-rotation relationship from the young Hyades and Praesepe (0.6 Gyr) to the old M 67 ( $4.5 \mathrm{Gyr})$ through the two intermediate age clusters IC 4651 and NGC $3680(\sim 1.7 \mathrm{Gyr})$. The full amplitude of the activity index within a cluster is slightly above $60 \%$ for all clusters but one, NGC 3680, in which only two stars were observed. As a comparison, the same Solar Ca II index varies by $\sim 40 \%$ during a solar cycle. Four of our clusters (Hyades and Praesepe, IC 4651 and NGC 3680) are pairs of twins as far as age is concerned; the Hyades have the same chromospheric-activity level as Praesepe, at odds with early claims based on X-ray observations. Both stars in NGC 3680 are indistinguishable, as far as chromospheric activity is concerned, from those in the coeval IC 4651. This is a validation of the existence of an age-activity relationship. On the other hand, the two intermediate age clusters have the same activity level as the much older M67 and the Sun. Our data therefore shows that a dramatic decrease in chromospheric activity takes place in solar stars between the Hyades and the IC 4651 age, of about 1 Gyr. Afterwards, activity remains virtually constant for more than 3 Gyr. We have also measured $v \sin i$ for all of our stars and the average rotational velocity shows the same trend as the chromospheric-activity index. We briefly investigate the impact of this result on the age determinations of field G dwarfs in the solar neighborhood; the two main conclusions are that a consistent group of "young" stars (i.e. as active as Hyades stars) is present, and that it is virtually impossible to give accurate chromospheric ages for stars older than $\sim 2$ Gyr. The observed abrupt decline in activity explains very well the Vaughan-Preston gap.
\end{abstract}

Key words. stars: late-type - stars: activity - stars: chromosphere - stars: rotation

\section{Introduction}

Chromospheric activity is powered by rotation in the presence of a deep convective envelope. Because of rotation braking by a magnetised stellar wind, rotational velocity decreases with age, as well as activity, unless the angular momentum is sustained by tidal interaction, as in the case of short-period binaries. This simple picture is at the basis of the so called ageactivity-rotation relationship in solar-type stars and forms the paradigm of stellar activity in the last 30 years. The literature on the argument is enormous and the reader may refer to a number of works, among which we mention the Parker's review on the solar magnetic fields (Parker 1970).

The present study aims at improving this picture by accurately ascertaining the form of the activity-age law on the basis of a new dataset. The decaying law of chromospheric activity

* Observations collected at the ESO VLT. Some data presented herein were obtained at the W. M. Keck Observatory, which is operated as a scientific partnership among the California Institute of Technology, the University of California, and the National Aeronautics and Space Administration. The Observatory was made possible by the generous financial support of the W. M. Keck Foundation. is a useful tool in determining ages of field main sequence stars and chromospheric ages have been used to undertake detailed studies of the star formation rate and chemical enrichment of the Galaxy (Rocha Pinto et al. 2000, Papers II, I).

The flux emission in the core of the $\mathrm{K}$ line of $\mathrm{Ca}$ II is the most effective tracer to probe the chromospheric activity in solar-type stars (see e.g., Blanco et al. 1974; Linsky \& Ayres 1978). Indeed, we intend to use in this study the equivalent width of the line emission core, corrected for the underlying stellar photospheric contribution and transformed into flux at the stellar surface $\left(F_{K}^{\prime}\right)$, as an index of the level of chromospheric activity (Pasquini et al. 1988).

Among similar indices the most used and widely known is the Mount Wilson $R_{H K}^{\prime}$, which is the flux in the Mount Wilson photometer passband normalized to the stellar effective temperature (Noyes et al. 1984). The apex indicates that the quantity is corrected for the the photospheric-flux contribution. This convention will be adopted throughout this paper. While the Mount Wilson index is a contrast index, our $F_{K}^{\prime}$ is an absolute flux. If we indicate the chromospheric flux of the $\mathrm{H}$ and $\mathrm{K}$ line cores at the stellar surface $\left(\mathrm{erg} \mathrm{cm}^{-2} \mathrm{~s}^{-1}\right)$ as $F_{H K}^{\prime}$, and the bolometric one as $F_{\mathrm{BOL}}$, then $R_{H K}^{\prime}$ is the ratio $\frac{F_{H K}^{\prime}}{F_{\mathrm{BOL}}}$. 
While chromospheric activity has been widely studied in field stars (see e.g., Henry et al. 1996; Wilson 1963; Pasquini et al. 1990; Pasquini 1992), relatively little work has been done on clusters, which are the perfect calibrators of the age-activityrotation relationship: they are in fact homogeneous samples of stars with well determined ages.

For the reasons explained in van den Bergh \& McClure (1980), old open clusters are rare and tend to be distant. This is why the original Skumanich (1972) law was based only on 1 point older than the Hyades, which was the Sun. Only with the advent of 4-m telescopes and high-sensitivity instruments has it been possible to acquire Ca II spectra for subgiants in M 67 (Dupree et al. 1999), which is as distant as $850 \mathrm{pc}$ (see e.g., Carraro et al. 1996). Nowadays 8-m class telescopes are sufficiently sensitive to allow the study of Ca II emission even in the dwarfs of distant clusters.

Barry et al. (1987) made the most consistent attempt to cover the lack of old-intermediate age clusters data. They built a 2- $\AA$ resolution system and used it to observe several intermediate and old clusters. This very interesting attempt, on the other hand, needs to be looked with some caution, since, for instance, the typical $\mathrm{G}$ star emission core is much larger than $2 \AA$. Therefore a 2- $\AA$ resolution is sensitive to other factors, such as zero point corrections and photospheric profile in addition to activity (see also Soderblom et al. 1991, for a discussion on Barry et al.'s data). A narrower, pure chromosphericactivity index based on high-resolution spectroscopy, such as the quoted $F_{K}^{\prime}$, is much more reliable. This is the basis of the present work.

\section{Observations and data sample}

Our study is based on spectra of 35 stars in 5 open clusters and on the solar data from White \& Livingston (1981). White and Livingston made a very detailed study of the Ca II solar K line covering the cycle 21 from the first minimum to the maximum. Because of differences in data analysis and reduction, their 1- $\AA$ indices have to be multiplied by 1.11 to be homogeneous with ours.

The Praesepe, NGC 3680, IC 4651 and M 67 observations were obtained with the UVES spectrograph at the VLT Kueyen telescope. The blue slit was set at 0.7 arc seconds giving a resolving power of $R \sim 60000$ (Dekker et al. 2000). For the faintest stars, up to 3 consecutive 90-min exposures were co added, in order to reach enough signal at the bottom of the deep Ca II K line which is typically less than $10 \%$ of the continuum in non active stars. The signal to noise ratio per pixel at the bottom of the $\mathrm{K}$ line ranges from about 10 for the worst spectra in M 67 to about 30 for most of the spectra in Praesepe.

The red slit was set to 0.4 arc seconds, to obtain a resolution of $R=100000$

The data were reduced using the UVES pipeline (Ballester et al. 2000), radial velocity corrected and finally co-added.

The Hyades spectra were obtained within the program of search for extra-solar planets among the solar-type stars of this cluster (Cochran et al. 2002; Paulson et al. 2002) with HIRES at the Keck Observatory. They have a resolution similar to the blue UVES ones ( $R=60000)$, and a signal to noise ratio at the bottom of the $\mathrm{K}$ line ranging from about 20 to about 30 . The original Hyades' sample comprises a large range of spectral types, and was selected to include only stars with $v \sin i$ lower than $15 \mathrm{~km} \mathrm{~s}^{-1}$. We have selected for the present study only the dwarfs with $B-V$ colours similar to the solar one. According to Strassmeier et al. (2000) (see the $g$ panel in their Fig. 4), latetype stars which rotate faster than $15 \mathrm{~km} \mathrm{~s}^{-1}$ are rare exceptions (unless we are dealing with clusters much younger than the Hyades, see Bouvier 1997), therefore we do not expect to have a strong bias towards slow rotators.

All the Ca II data have been normalized to the $3950.5 \AA$ pseudocontinuum (Catalano 1978).

The clusters span a wide range of ages, from the young Hyades and Praesepe (0.6 Gyr) to the old M 67 (4.8 Gyr). Together with the Sun, they constitute three pairs of objects at three different ages: Hyades and Praesepe, IC 4651 and NGC 3680 (1.6 and 1.8 Gyr), and M67 and the Sun (4.8 and $4.48 \mathrm{Gyr}$ ). This allows to have a first check on the consistency of the age-activity-rotation relationship: if each pair has the same chromospheric-activity level, we will have a strong confirmation that chromospheric activity is an agedriven parameter.

In addition, the comparison between Hyades and Praesepe is extremely interesting, after the finding by Randich \& Schmitt (1995) that the solar stars belonging to these clusters have different X-ray (coronal) emission, with the Hyades showing substantially higher X-ray luminosities than Praesepe (see Barrado y Navascues et al. 1997, and reference therein).

The ages of M 67, NGC 3680 and IC 4651 are taken from Carraro \& Chiosi (1994). Hyades and Praesepe, with some differences between various authors, are often thought to be coeval with ages of about $0.6 \mathrm{Gyr}$ (see e.g., Perryman et al. 1998; Mermilliod 1981; Allen 1973). For the solar age we have used Guenther's work (1989), based on the age of the oldest meteorites.

All the stars observed with the VLT were chosen to be on the main sequence, high-probability members of the clusters and not known to be binaries at the moment of the observation. The choice was done using as references Nordström et al. (1996) for NGC 3680, Meibom et al. (2002) for IC4651 and several sources for M 67, including Latham et al. (1992) for the binary determination in this cluster. Our stars have colours comprised within $0.51<(B-V)_{0}<0.73$, closely encompassing the solar colour which, with some variations between different authors, is evaluated around $B-V=0.63$ (see e.g., Straizis \& Valiuga 1994). We also note that the paucity of stars observed in NGC 3680 is imposed by the fact that, as shown by Nordström et al. (1997), this cluster has very few single G stars members left, most of its low mass members being dispersed in its lifetime.

As far as Praesepe is concerned, out of the 7 stars observed we chose 3 stars detected in X-ray by Randich \& Schmitt (1995) and 4 undetected, in order to check whether the chromospheric activity was different between the two groups, as expected if chromospheric and coronal activity were strongly correlated.

All the observed stars are summarized in Table 1. Star names are taken from Eggen (1969) for NGC 3680, 
Table 1. The measurements of our sample stars. The names in Col. 1 are taken from Eggen (1969) for NGC 3680, Anthony Twarog et al. (1988) (AMC) and Eggen (1971) for IC 4651, Sanders (1977) for M 67, Klein Wassink (1927) for Praesepe \& van Bueren (1952) for the Hyades stars.The photometry is taken from the reference papers for IC 4651, from Nordström et al. (1997) for NGC 3680, from Hipparcos Catalogue (ESA 1997) for Hyades, from Jones \& Stauffer (1991) for Praesepe, with the exception of KW 368 which is taken from Jones \& Cudworth (1983), and from Montgomery et al. (1993) for M 67. Regarding chromospheric-activity indices measurements, there are the 1indices derived from spectra either non corrected (Col. 4) or corrected (Col. 5) for the absorption of the IS medium. From the corrected index we have subtracted the photospheric contribution, which is $0.049 \AA$, and transformed the result into flux. The final data are in Col. 6 . $v$ sin $i$ measurements in Col. 8 are taken from Paulson et al. (2003) for Hyades, for all the other clusters are based on the FWHMs in Col. 7 via the calibrations in Sect. 3.2.

\begin{tabular}{|c|c|c|c|c|c|c|c|}
\hline STAR & [mag] & $\begin{array}{c}(B-V)_{0} \\
{[\mathrm{mag}]}\end{array}$ & $\begin{array}{c}1-\AA \text { index } \\
\text { non ISM corr. } \\
\AA\end{array}$ & $\begin{array}{c}1-\AA \text { index } \\
\text { ISM corr. } \\
\AA\end{array}$ & $\begin{array}{c}F_{K}^{\prime} \\
\text { ISM corr. } \\
10^{6}\left[\mathrm{erg} \mathrm{cm}^{-2} \mathrm{~s}^{-1}\right]\end{array}$ & [pixels] & $\begin{array}{c}v \sin i \\
{\left[\mathrm{~km} \mathrm{~s}^{-1}\right]}\end{array}$ \\
\hline \multicolumn{8}{|c|}{ PRAESEPE } \\
\hline KW 49 & 10.65 & 0.59 & 0.219 & 0.219 & 2.10 & 14.95 & 8.57 \\
\hline KW 100 & 10.57 & 0.51 & 0.238 & 0.238 & 3.03 & 20.27 & 13.06 \\
\hline KW 326 & 11.20 & 0.56 & 0.220 & 0.220 & 2.35 & 11.54 & 5.15 \\
\hline KW 368 & 11.30 & 0.71 & 0.258 & 0.258 & 1.59 & 10.91 & 4.39 \\
\hline KW 392 & 10.78 & 0.51 & 0.231 & 0.231 & 2.92 & 12.09 & 5.77 \\
\hline KW 418 & 10.51 & 0.53 & 0.219 & 0.219 & 2.57 & 13.12 & 6.84 \\
\hline \multicolumn{8}{|c|}{ HYADES } \\
\hline van Bueren 1 & 7.38 & 0.567 & 0.165 & 0.165 & 1.55 & 12.46 & 5.5 \\
\hline van Bueren 2 & 7.78 & 0.617 & 0.208 & 0.208 & 1.78 & 12.65 & 5.5 \\
\hline van Bueren 10 & 7.85 & 0.593 & 0.266 & 0.266 & 2.66 & 13.04 & 6.2 \\
\hline van Bueren 15 & 8.05 & 0.657 & 0.293 & 0.293 & 2.34 & 12.60 & 5.4 \\
\hline van Bueren 17 & 8.45 & 0.693 & 0.257 & 0.257 & 1.71 & 12.10 & 4.5 \\
\hline van Bueren 18 & 8.05 & 0.640 & 0.279 & 0.279 & 2.36 & 12.46 & 5.4 \\
\hline van Bueren 31 & 7.46 & 0.560 & 0.246 & 0.246 & 2.70 & 16.39 & 10.0 \\
\hline van Bueren 49 & 8.22 & 0.599 & 0.192 & 0.192 & 1.71 & 11.28 & 2.8 \\
\hline van Bueren 52 & 7.79 & 0.599 & 0.233 & 0.233 & 2.20 & 13.12 & 6.5 \\
\hline van Bueren 65 & 7.41 & 0.537 & 0.187 & 0.187 & 2.04 & 15.42 & 8.8 \\
\hline van Bueren 66 & 7.49 & 0.560 & 0.238 & 0.238 & 2.59 & 14.88 & 8.6 \\
\hline van Bueren 73 & 7.83 & 0.609 & 0.255 & 0.255 & 2.38 & 13.35 & 6.8 \\
\hline van Bueren 88 & 7.78 & 0.550 & 0.213 & 0.213 & 2.32 & 11.68 & 1.0 \\
\hline van Bueren 97 & 7.90 & 0.631 & 0.264 & 0.264 & 2.28 & 12.70 & 5.4 \\
\hline van Bueren 118 & 7.72 & 0.578 & 0.146 & 0.146 & 1.25 & 12.55 & 5.3 \\
\hline \multicolumn{8}{|c|}{ IC 4651} \\
\hline AMC 1109 & 14.534 & 0.599 & 0.093 & 0.102 & 0.63 & 10.20 & 3.39 \\
\hline AMC 2207 & 14.527 & 0.595 & 0.073 & 0.085 & 0.44 & 10.03 & 3.11 \\
\hline AMC 4220 & 14.955 & 0.695 & 0.113 & 0.133 & 0.68 & 9.96 & 2.99 \\
\hline AMC 4226 & 14.645 & 0.624 & 0.079 & 0.093 & 0.48 & 9.78 & 2.67 \\
\hline Eggen 45 & 14.27 & 0.53 & 0.068 & 0.074 & 0.37 & 10.26 & 3.48 \\
\hline \multicolumn{8}{|c|}{ NGC 3680} \\
\hline Eggen 60 & 14.290 & 0.641 & 0.090 & 0.095 & 0.47 & 9.88 & 2.85 \\
\hline Eggen 70 & 14.589 & 0.651 & 0.075 & 0.084 & 0.34 & 10.49 & 3.82 \\
\hline \multicolumn{8}{|c|}{ M 67} \\
\hline Sanders 746 & 14.380 & 0.659 & 0.091 & 0.108 & 0.56 & 9.45 & 1.96 \\
\hline Sanders 1012 & 14.516 & 0.687 & 0.116 & 0.133 & 0.71 & 9.66 & 2.43 \\
\hline Sanders 1048 & 14.411 & 0.635 & 0.073 & 0.085 & 0.37 & 9.62 & 2.35 \\
\hline Sanders 1092 & 13.308 & 0.581 & 0.074 & 0.088 & 0.50 & 10.99 & 4.49 \\
\hline Sanders 1255 & 14.486 & 0.616 & 0.086 & 0.105 & 0.63 & 9.80 & 2.71 \\
\hline Sanders 1283 & 14.115 & 0.590 & 0.067 & 0.082 & 0.41 & 10.25 & 3.46 \\
\hline Sanders 1287 & 14.030 & 0.558 & 0.079 & 0.090 & 0.56 & 10.39 & 3.67 \\
\hline
\end{tabular}


Anthony Twarog et al. (1988) and Eggen (1971) for IC 4651, Sanders (1977) for M 67, Klein Wassink (1927) for Praesepe and van Bueren (1952) for the Hyades stars. The photometry is taken from the reference papers for IC 4651, from Nordström et al. (1997) for NGC 3680, from Hipparcos Catalogue (ESA 1997) for Hyades, from Jones \& Stauffer (1991) for Praesepe, with the exception of KW 368 which is taken from Jones \& Cudworth (1983), and from Montgomery et al. (1993) for M 67. For Hyades no correction at all for interstellar (IS) extinction is applied (Taylor \& Joner 2002), and NGC 3680 colours are given already dereddened by Nordström et al. (1997). $B-V$ colours of Praespe, M 67 and IC 4651 had to be corrected for colour excess. We used $E(B-V)=0.05$ for M 67 (also taken from Montgomery et al. 1993), $E(B-V)=0.086$ for IC 4651 (Anthony Twarog \& Twarog 1987) and $E(B-V)=$ 0.02 for Praesepe (Taylor \& Joner 2002). The uncertainties in reddening are not at all critical for the analysis performed.

The photometric data retrieved from the literature are shown in the second and third columns of Table 1 ( $V$ magnitude and $B-V$ colour index, respectively). The different chromospheric-activity indices are reported in Table 1 from the fourth to the sixth column and explained in the caption.

The quantity shown in the seventh Column is the Full Width Half Maximum ( $F W H M)$ of the cross correlation profile of the spectra. The cross correlation profiles were obtained as described in Melo et al. (2001): we used as template a box-shaped mask, suitable for solar-type stars, which was kindly provided by Claudio Melo. We processed the red part of the red arm UVES-spectra, all rebinned at the same step $(0.0174087 \AA)$. The $1 \sigma$ uncertainties of the FWHMs are of about $1 \%$.

The rotational velocities determined by us are given in the last column . They are obtained from the FWHMs via the calibrations of Sect. 3.2.

It is worth noticing that what really matters for the present purpose is the evolution of the angular momentum of the stars, we are therefore mostly interested in the differential $v \sin i$ values.

\section{Analysis}

\subsection{Ca ll fluxes}

The analysis is based on the measurement of flux in the emission core of the Ca II K line. This line is the most sensitive indicator of chromospheric activity, and it has been widely studied in a number of different environments, including the Sun and solar stars (Linsky \& Avrett 1970; Linsky et al. 1979; Pasquini et al. 1988; Pasquini 1992; White \& Livingston 1981).

In general, the most sensitive area is that comprised between the line K1 minima, but those are hardly seen in low activity stars or in intermediate $\mathrm{S} / \mathrm{N}$ ratio spectra.

A very useful index of the level of chromospheric activity is the area of the $1-\AA$ wide band centered on the line core (hereafter referred to as $1-\AA$ index). This $1-\AA$ band corresponds roughly to the separation between the $\mathrm{K}_{1}$ minima for an active star (as those in Hyades or Praesepe), and it is about $30 \%$ larger than the $\mathrm{K}_{1}$ minima separation for a star as active as the Sun (White \& Livingston 1981; Pasquini 1992). The 1-Å index was measured in the spectra either uncorrected or corrected for IS absorption. The results are shown in the fourth and fifth column of Table 1 respectively. In addition, we recall that the $F_{K}^{\prime}$ is a measurement of a contrast between the chromospheric emission and the underlying photospheric flux. Several calibrations can be used to eliminate this offset and to quantify different bandwidths (Linsky et al. 1979); this is widely discussed (e.g. in Pasquini et al. 1988), and will not be repeated here.

Although our clusters are among the closest and less reddened, the Ca II lines could be contaminated by the IS Ca II, a possibility that we have taken into account as explained later in this section.

We summarize the different steps required to get the final fluxes from the observed spectra.

\section{Spectra normalization and rectification \\ 2. Subtraction of the IS line \\ 3. Index measurement \\ 4. Subtraction of the photospheric contribution \\ 5. Calibration in Fluxes.}

The observed spectra have been normalized to the $3950.5 \AA$ pseudocontinuum, as done in Catalano (1978) and then in Pasquini et al. (1988). Since the spectra are the result of a merging of different echelle orders, we must check that merging is properly made. We did this in two ways: by observing early-type stars (i.e. stars with no Ca II K stellar line), and by comparing the target spectra with a synthetic solar spectrum (courtesy of P. Bonifacio). This comparison of observed with photospheric-line profiles has been largely used by us, not only to verify that the rectification of the spectra was properly done, but also to check that other effects, such as background light, were correctly taken into account. The $\mathrm{K}$ line is very suitable for this purpose, because it is very deep, and therefore sensitive to small mistakes in setting the zero flux level. In Fig. 1 we show the average spectra of all the cluster stars and the solar one, all plotted over the synthetic solar CaII K stellar line. The solar spectrum is taken from the UVES archive ${ }^{1}$. We checked that the observed photospheric-line profiles successfully overlap after normalization. This is only possible because all of the stars have similar colour to that of the Sun. We have also checked the consistency of each observed $\mathrm{K}$ line profile with the synthetic solar K line profile. The spectra of the single stars are shown in Figs. 2 and 3.

For all clusters only one component of the IS Ca II K line falls in the stellar-spectra region where the $1-\AA$ index is measured. In order to evaluate its contribution, we have observed for each of the 3 reddened clusters a reference star with an easily recognisable IS component. These stars show no narrow stellar line inside the Ca II absorption feature either because they are fast rotators (as in the case of Sanders 1082 in M 67, an Algool-type binary, and Eggen 70 in IC 4651) or because they are hot enough (as in the case of Eggen 10 in NGC 3680) to present a pronounced broad deep core. In Fig. 4, upper panels, the spectra of the quoted reference stars are shown. For each of them, the spectrum without the IS contribution is plotted over

\footnotetext{
1 http://www . eso.org/observing/dfo/quality/UVES/ pipeline/
} 


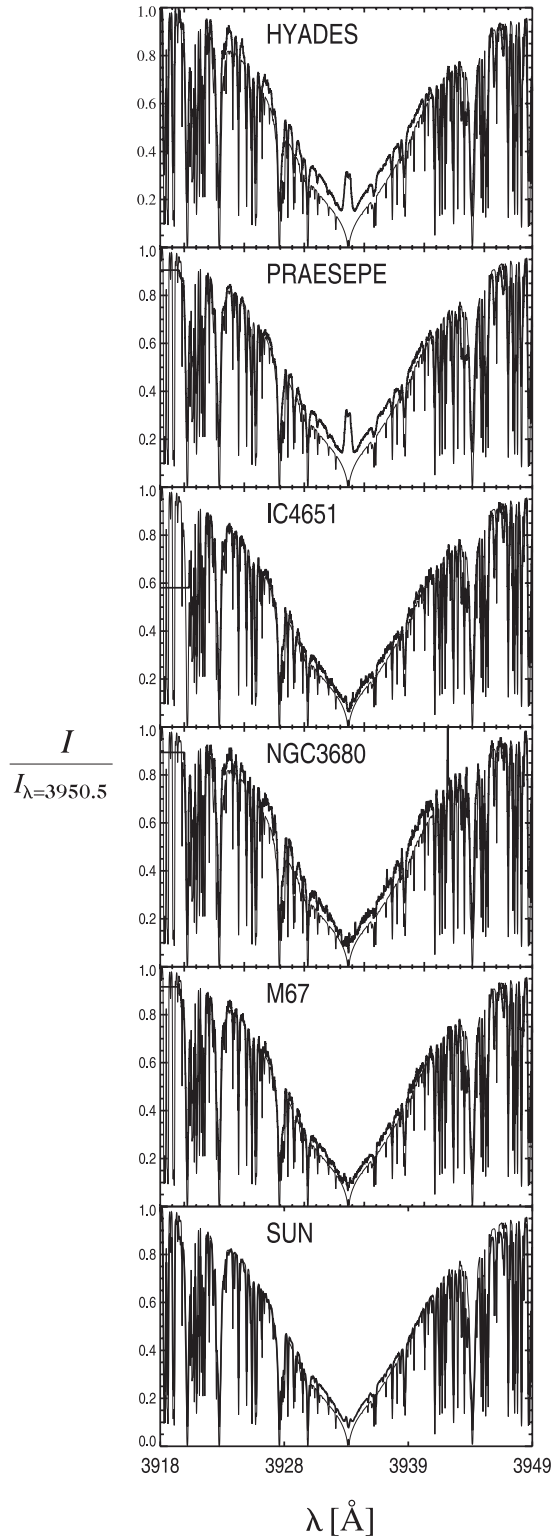

Fig. 1. Average clusters Ca II K line spectra. The synthetic photospheric-solar model is super-imposed. In the bottom panel we plot the real solar spectrum, also super-imposed on its model. The effect of the back heating of the chromosphere is also evident outside the core.

the original one. The latter has been divided by the former, and the result is the IS contribution, which is shown in the bottom panel of Fig. 4.

Eggen 10 is quite hot, but this is not the case for Sanders 1082 and Eggen 70. If they were slightly active, their emission would partially fill in the absorption IS line. In this case, the IS negative contribution would be underestimated by the removal procedure. We are, however, confident that our estimate is quite robust, because Sanders 1082 and Eggen 70 are rather fast rotators, therefore if their chromospheric emission were relevant, they would be as broad as the photospheric lines and the emission wings would be visible outside the IS contribution.
Each $\mathrm{K}$ line spectrum of our target stars has been divided by the appropriate IS spectrum, and the $1-\AA$ indices measured again. The results are given in the fourth column of Table 1. In Fig. 5 we plot the $F_{K}^{\prime}$ vs. $B-V$ before and after the IS-line contribution has been subtracted (respectively left and right panels). Clearly the effect of this subtraction, although noticeable, does not affect dramatically the relative emission between the different clusters, which is not surprising, given the low reddening of the clusters.

Chromospheric fluxes decrease for cooler stars (see e.g., Soderblom et al. 1991). In Fig. 5 such a trend is not present, with the possible exception of Praesepe stars. By comparing our plot with that of Soderblom et al. (1991, see Fig. 6 therein), we do not expect our results to be significantly affected by the different colour distribution of the targets in the different clusters.

We have subtracted from the $1-\AA$ indices the photospheric contribution, calculated in the synthetic photospheric-solar model. The quantity subtracted amounts to $0.049 \AA$. Note that, even though this subtraction is the same for all of the stars, once the $1-\AA$ indices have been transformed into fluxes in the way explained later in this section, the photospheric contribution becomes colour dependent, because the transformation into flux is colour dependent. The photospheric contribution we subtracted does not differ more than $20 \%$ from other approximations, e.g. the one used by Noyes et al. (1984), and Duncan et al. (1984). The choice of another approximation does not significantly change the result, even though it might affect the spread within each cluster.

We have derived the chromospheric fluxes at the stellar surface transforming the IS corrected, photospheric subtracted equivalent widths into flux densities at the stellar surface ( $\mathrm{erg} \mathrm{s}^{-1} \mathrm{~cm}^{-2}$ ). This was made in three steps. We first computed the $V-R$ colours from the $B-V$ ones. We used the calibration in Pasquini (1985):

$V-R=0.618 \cdot(B-V)^{2}-0.183 \cdot(B-V)+0.375$.

We have then used the calibration in Pasquini et al. (1988) between the $V-R$ colour and the flux at the stellar surface of the pseudo-continuum radiation at $3950.5 \AA$ :

$\log F_{3950.5}=8.459-2.833 \cdot(V-R)$.

We have finally multiplied the equivalent widths for $F_{3950.5}$, obtaining the fluxes in the sixth column of Table 1.

We need to estimate the absolute and relative accuracy of the indices and of the fluxes. Actually, the relative uncertainties are the most relevant here, since we will ultimately compare the ratio of the fluxes between the different clusters. The absolute value, although interesting, is just a normalised value for the purpose of the age-activity law.

The Signal to Noise per pixel is not the limiting factor of the accuracy, even though it is only of $\sim 10$ in the spectra of the faintest stars, because we integrated on $1 \AA$, namely $\sim 48$ pixels and $\sim 37$ for HIRES spectra). For the intermediate age clusters and M 67, the dominant sources of error in the measurements could come from an incorrect treatment of the inter-order (and scattered) light or from rectification problems. As we have 


\section{HYADES}

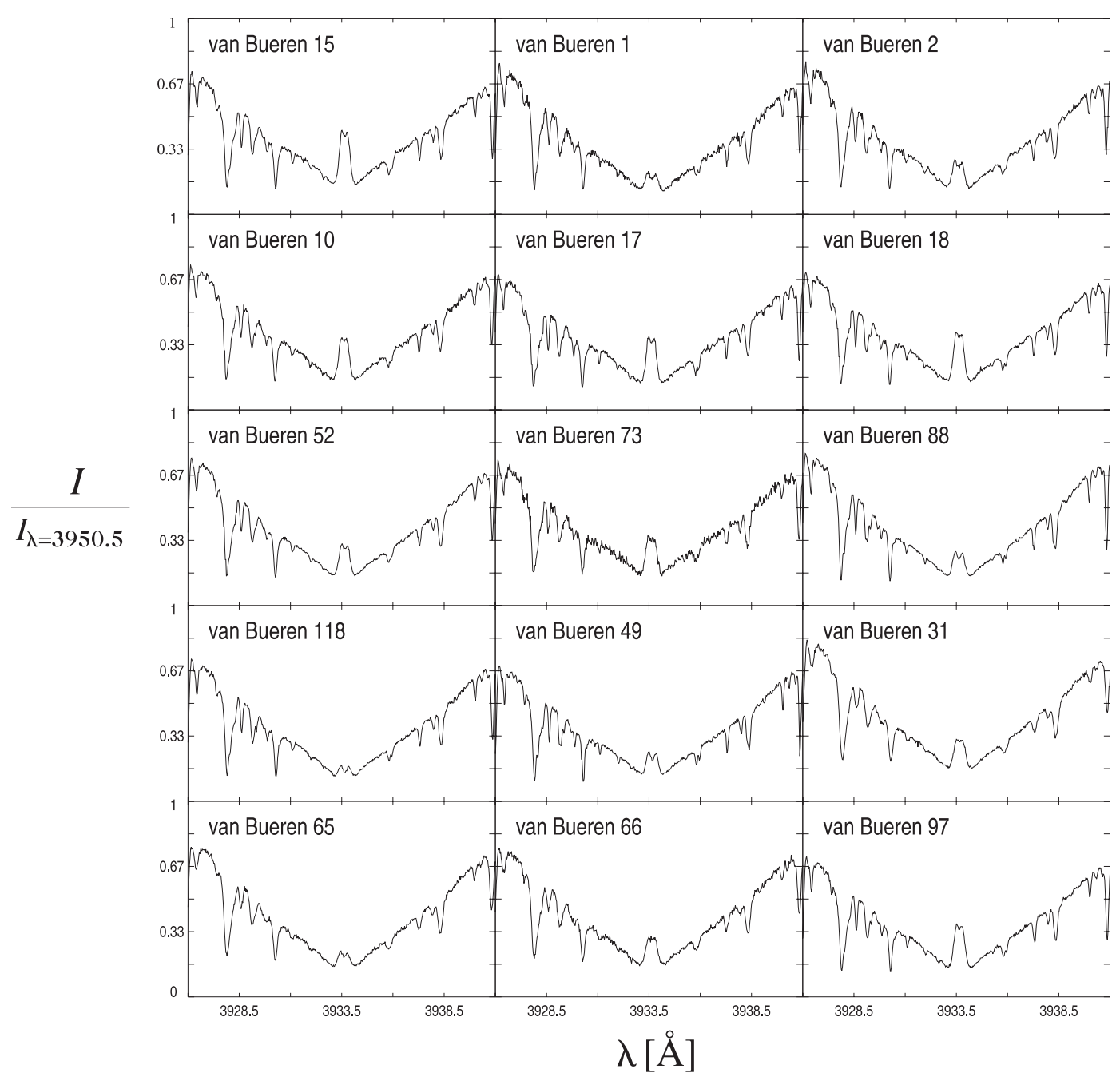

Fig. 2. HIRES spectra of the Hyades stars of our sample.

mentioned before, we think we are rather free of these problems, because of the satisfactory match between the solar synthetic and stellar observed profiles of the $\mathrm{K}$ line wings (i.e. the region between $\sim 3920$ and $\sim 3950 \AA$, outside the line core). We have first divided the photospheric spectra of all the stars of each cluster by their average spectrum, finding peak to peak differences of less than $7 \%$ in their profile ratios. We have then repeated the same comparison with the solar synthetic spectrum; and the differences between the stellar spectra and the solar model are greater. They are up to $20 \%$ for Hyades and Preasepe stars, while they are of up to about $10 \%$ for the other clusters. Hyades and Praesepe stars show exactly the same behaviour, despite that the spectra were obtained with two different spectrographs. The stellar spectra always have higher profiles than the solar model and that is true even for the Sun itself (cf. Fig. 1). This can be due to a low level of residual scattered light in the spectrograph, but the main reason is that the solar model we used does not include any chromospheric effect, which will tend to heat the higher layers of the photosphere and therefore to raise the photospheric profiles as well. This effect is well known at least in the Sun, where the differences between the quiet and plage regions arise also in the photospheric wings of the K line (La Bonte 1986, see Fig. 1 therein).

Since the IS correction could be rather tricky, we have tried several different fits of the IS-line wings finding only minor variations and we estimate the error from a wrong computation of the IS line as negligible. It is important also to see that this error is the same in both absolute and relative terms and that the whole IS correction accounts for at most $20 \%$ of the chromospheric flux measured.

The errors in $F_{K}^{\prime}$ come from three main sources:

- the uncertainty in the equivalent width measurement, which in turn has, as mentioned, many other causes;

- uncertainty in the $V-R$ vs. $B-V$ calibration;

- the uncertainty in the $B-V$ colours.

The absolute flux determination has an error of $30 \%$, due to the uncertainties in the $\log F_{3950.5}$ vs. $(V-R)$ calibration. However this affects mainly the zero point of our $F_{K}^{\prime}$ measurements, and only to a much smaller extent the relative comparison. 
PRAESEPE

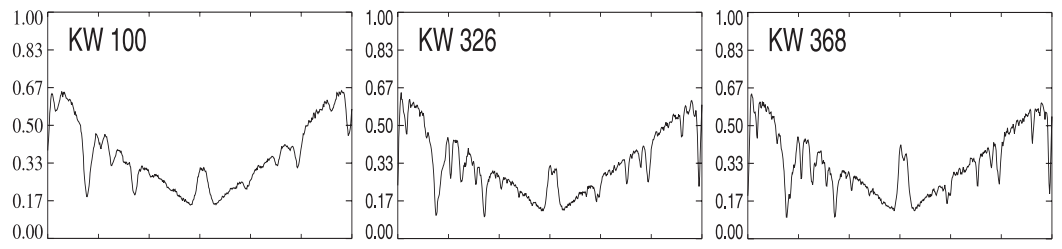

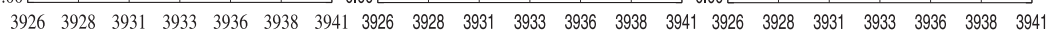

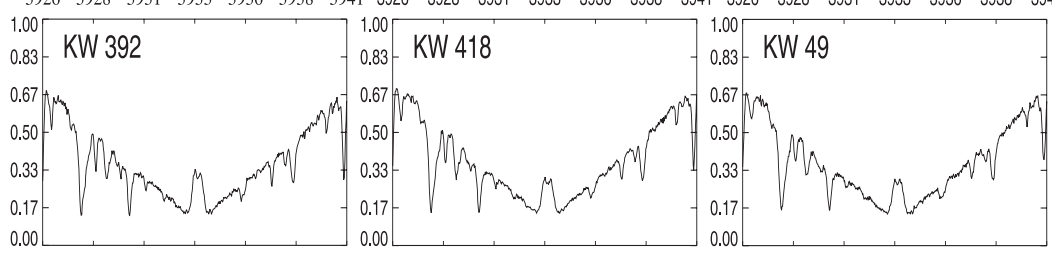

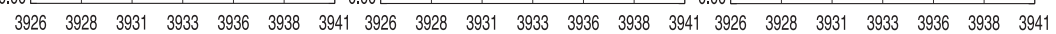

IC 4651
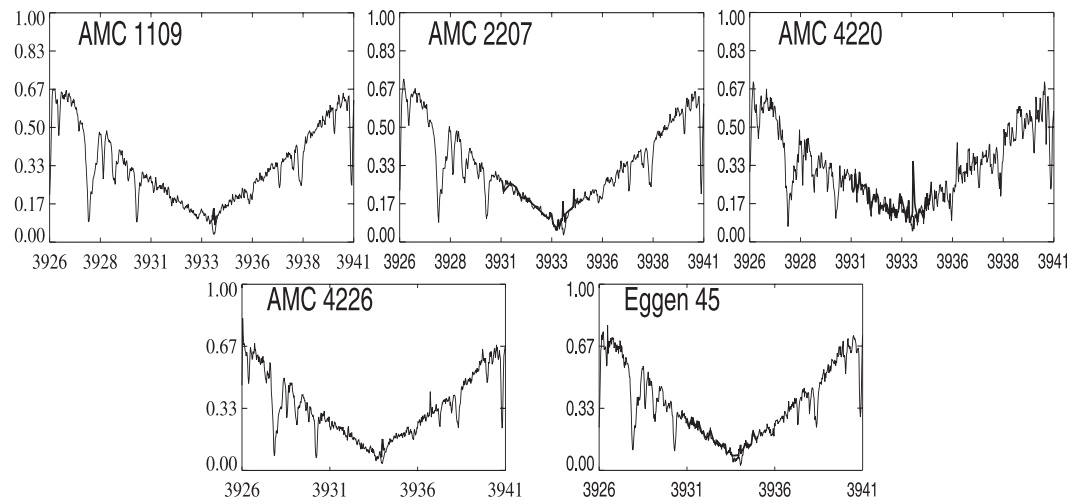

$\frac{I}{I_{\lambda=3950.5}}$

NGC 3680
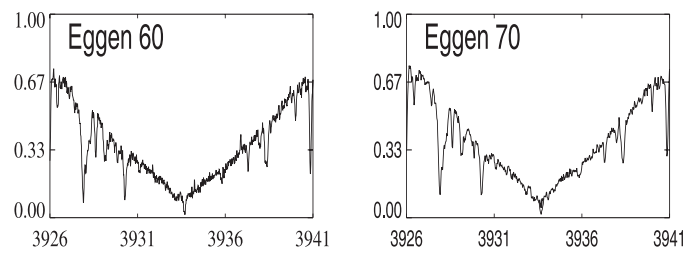

M 67
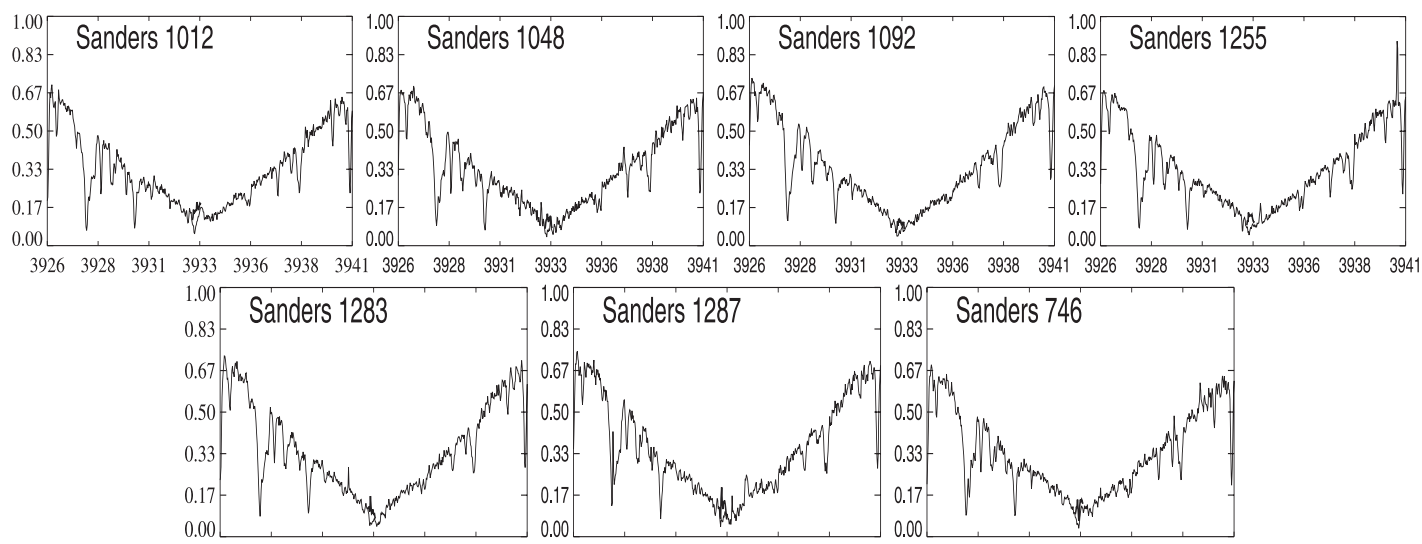

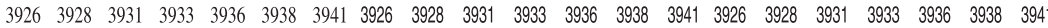

$\lambda[\AA]$

Fig. 3. UVES spectra of the stars of our sample. For the stars in IC 4651, NGC 3680 and M 67, in each panel, the spectrum non corrected for IS line is plotted over the corrected one. 

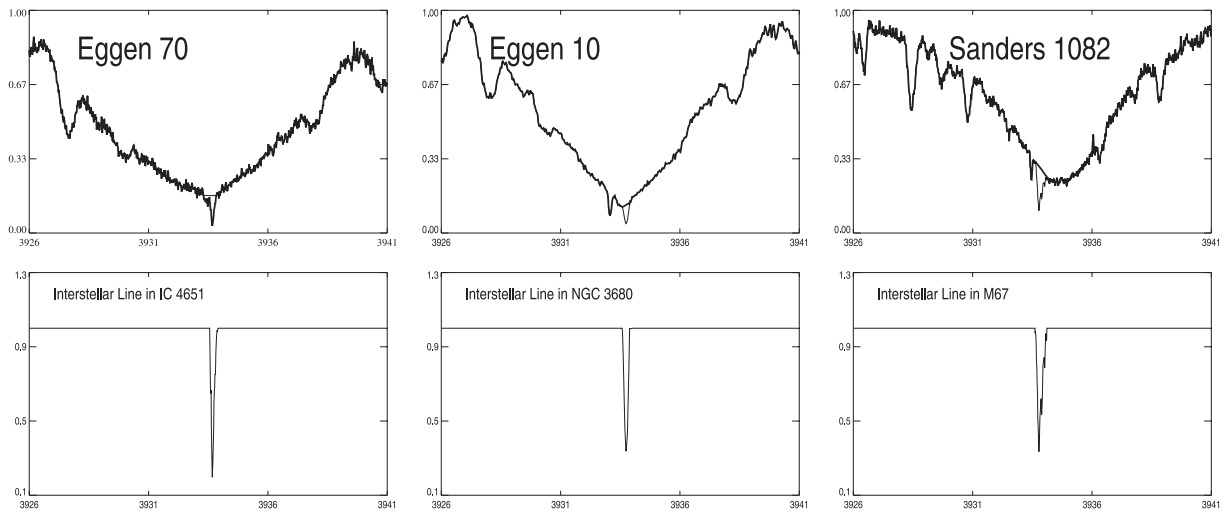

Fig. 4. Template spectra used to detect the IS-line feature. The original spectra (one for each of the 3 distant clusters) are over plotted on the supposed non contaminated profile on the 3 upper panels. In the lower panels the resulting IS features are shown.
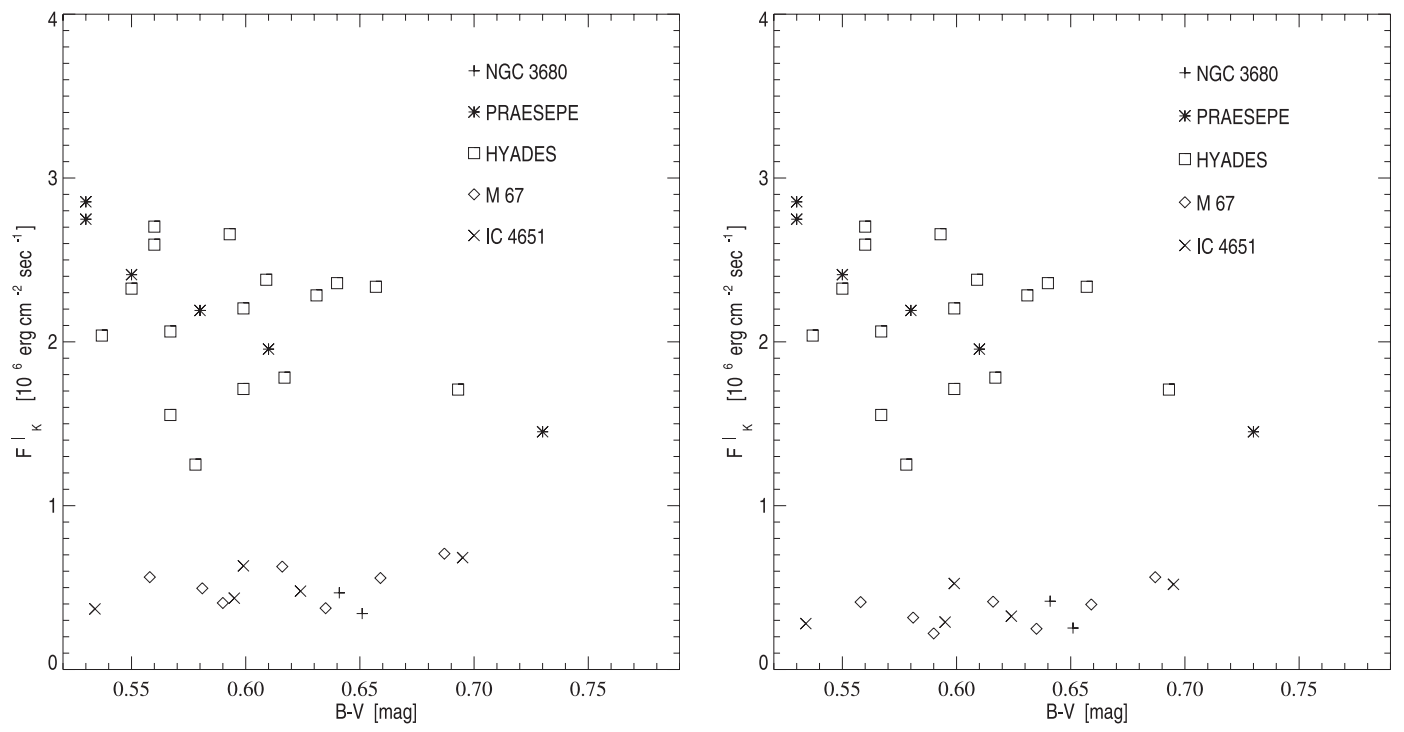

Fig. 5. The trend of $F_{K}^{\prime}$ with the colour. In the right panel the chromospheric activity is not corrected for the IS absorption, in the left one chromospheric-activity indices regarding IC 4651, NGC 3680 and M 67 stars are measured on IS absorption corrected spectra. There is no remarkable difference between the two diagrams. A decreasing trend with colour for chromospheric-activity index can only be seen for Praesepe stars, since the range of colours involved is narrow.

The two other sources of error give a contribution to the relative uncertainty $\frac{\Delta F_{K}^{\prime}}{F_{K}^{\prime}}$ that can be calculated on the basis of Eqs. (1) and (2). They are, respectively:

\section{$\frac{\Delta E W}{E W}$}

and

$(8.05 \cdot(B-V)-1.19) \cdot \Delta(B-V)$.

The typical relative maximum error in the equivalent width measurement of the single spectrum is within $10 \%$. For an error in the colour $\Delta(B-V)=0.04$, which we adopt as value of the maximum error in the colour, the corresponding relative error in the flux is, depending on $B-V$, between the 12 and the $18 \%$. If we add quadratically the two contributions, we finally obtain a maximum error in the single star measurement ranging from the 15 to the $20 \%$, if only relative values are concerned.

\subsection{Determination of the $v \sin i$}

As stated in Sect. 2, we have measured the $F W H M$ of the cross correlation profile for all the spectra of our sample, and the data are shown in the seventh column of Table 1.

Line broadening, as extensively discussed e.g. in Gray (1992), is caused by several effects - such as thermal motions, micro- and macro-turbulence, Stark effect, Zeeman effect, natural broadening, instrumental response and rotational velocity. We make two assumptions. The first is that the sum of these effects produces a Gaussian shape, which is a reasonable assumption for solar-type stars (see e.g. Pasquini et al. 1988). The second is that the difference in broadening between the stars of our sample is due only to rotation, or that the quoted effects contribute equally to the line broadening for all the stars, which is well justified given the similarity in the spectral type of the targets. We can now write:

$\sigma_{\mathrm{CCP}}=\sqrt{\sigma_{v \sin i}{ }^{2}+\sigma_{0}{ }^{2}}$ 
Table 2. Literature data used for the calibration in Fig. 6. The range of $B-V$ colours is $0.514-0.641$, closely encompassing the solar one.

\begin{tabular}{cccc}
\hline \hline STAR & Cluster & $v \sin i\left[\mathrm{~km} \mathrm{~s}^{-1}\right]$ & $F W H M$ [pixels] \\
\hline AT 1228 & IC 4651 & 15.7 & 22.24 \\
Eggen 15 & IC 4651 & 10.0 & 16.65 \\
Eggen 34 & IC 4651 & 25.2 & 37.18 \\
Eggen 45 & IC 4651 & 4.2 & 10.27 \\
Eggen 79 & IC 4651 & 21.8 & 31.42 \\
Eggen 99 & IC 4651 & 28.1 & 40.45 \\
Eggen 60 & NGC 3680 & 1.9 & 9.89 \\
Sun & - & 2.0 & 9.41 \\
\hline
\end{tabular}

where $\sigma_{\mathrm{CCP}}$ is the $\sigma$ the cross correlation profile, provided that it is a Gaussian. For the reasons explained above, $\sigma_{0}$ is expected not to change significantly from star to star, within our sample. Since $\sigma_{\mathrm{CCP}}$ and $\sigma_{v \sin i}$ are proportional respectively to the FWHM of the cross correlation profile and to $v \sin i$, we expect $v \sin i$ to be:

$v \sin i=A \cdot \sqrt{F W H M^{2}-B^{2}}$

where $A$ and $B$, like $\sigma_{0}$, are constants. Using $v \sin i$ measurements from the literature we can calibrate $A$ and $B$ in the previous relationship, and then calculate $v \sin i$ for all the stars. We have done this using the data of Table 2, in particular the $v \sin i$ measurements in the third column and the FWHMs in the fourth column.

Most of the spectra of the calibrators have been obtained in a parallel project on IC 4651 (Pasquini et al. 2003). The spectrum of the Sun is from the UVES archive, and is the same plotted in the bottom panel of Fig. 1. For Eggen 60 we used the FWHM of Table 1, since it is one of the targets. All the spectra used for the calibration are taken with the same instrument (UVES) at the same resolution $(R=100000)$ of our observations and the same spectral range is used to get the cross correlation profiles. The $v \sin i$ are taken from Meibom et al. (2002) for the stars in IC 4651 and from Nordström et al. (1996) for Eggen 60. For the Sun we adopted $2 \mathrm{~km} \mathrm{~s}^{-1}$.

For the calibration we decided not to use Eggen 70 in NGC 3680, even though for this $\operatorname{star} v \sin i$ is available. The reason is that the $1 \sigma$ error given for $v \sin i$ is three times larger than $v \sin i$ itself, that is $1 \mathrm{~km} \mathrm{~s}^{-1}$.

The calibration is a $\chi^{2}$ fit to the data of the Eq. (3). The root mean square of the residuals of the data around the fitting curve is $0.26 \mathrm{~km} \mathrm{~s}^{-1}$, no star deviates more than $10 \%$ of its $v \sin i$, and 5 out of 8 calibration stars deviate less than $2 \%$.

The result of the calibration is:

$v \sin i=0.72 \cdot\left(F W H M^{2}-9.05^{2}\right)^{1 / 2}$.

The curve is plotted in Fig. 6.

We have used it to compute the values given in the eighth column of Table 1 . We remind the eventual users that we used the spectral range $588-680 \mathrm{~nm}$, a spectral resolution of $R=100000$, and a rebinning step of $0.0174 \AA$

We note that the small residuals from our $\chi^{2}$-fit possibly overestimates its real accuracy. The reason is that our calibration is a secondary calibration, because also the calibrating

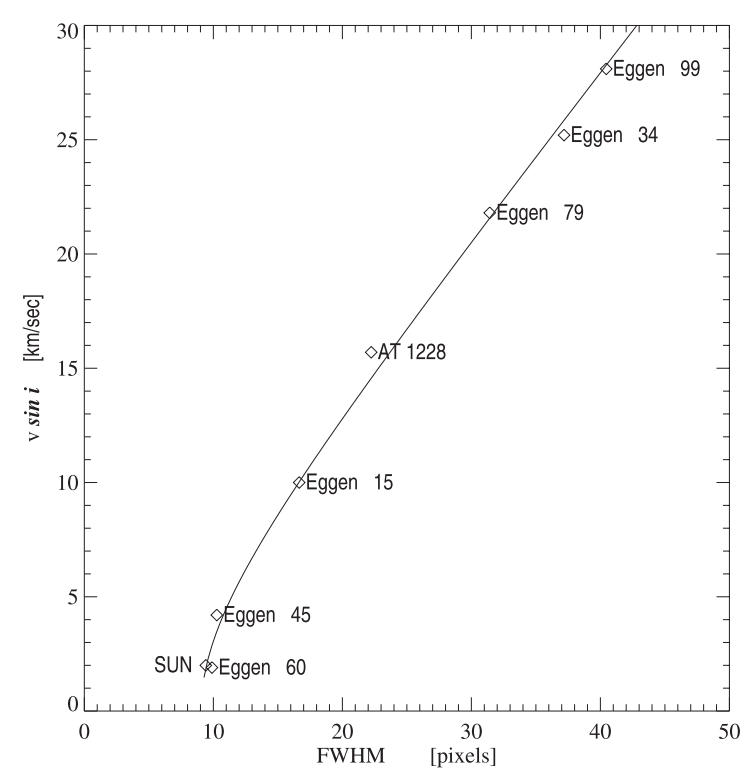

Fig. 6. Calibration of $v \sin i$ with the $F W H M$ of the cross correlation profiles of UVES spectra.

measurements are based on the FWHM of the cross correlation profiles. The errors may be quite larger in particular below $v \sin i \sim 3 \mathrm{~km} \mathrm{~s}^{-1}$, where other causes of line broadening become more important. We underline that we are mainly interested in differential values of $v \sin i$, that are as reliable as the root mean square of our calibration indicates.

Only for Hyades stars could we not use this calibration, since their spectra were taken with another spectrograph (HIRES) at a different resolution. Anyway, all of the $v \sin i$ for our Hyades targets are measured in Paulson et al. (2003). In this work, the rotational velocities are determined by comparing five synthetic Fe I lines with the observed ones, therefore temperature is taken into account. In Fig. 7 we plotted the diagram $v \sin i$ vs. FWHM. As for the calibration of UVES spectra, we computed a fit of an equation of the type of Eq. (3) to the data points. The result is that only one star deviates from the fit more than $5 \%$ of its $v \sin i$, and the median deviation is of the $2 \%$. This proves that, for targets comprised in the short $B-V$ colour range $0.51-0.73$, the $F W H M$ is virtually completely determined by $v \sin i$. The presence of an outlier, namely van Bueren 88 , confirms that more care has to be exercised in dealing with slow rotators. We will not use the outlier value of $v \sin i$ in the analysis of the angular momentum evolution. On the other hand, the overall quality of the data is evident when comparing Paulson et al.'s $v \sin i$ values with equatorial velocities from rotational periods.

Radick et al. (1987) provide precise period determination of 5 of our targets. They used narrow band by photometry to obtain light curves of 24 Hyades members. From the light curves it is possible to obtain the rotational period of the stars because rotational modulation of photospheric features (spots) induces photometric variations. We transformed the periods into rotational velocities, and found the data in Table 3 . We had to estimate the stellar radii, which were computed using $T_{\text {eff }}$ and computed bolometric luminosity. We transformed the $B-V$ colours into $T_{\text {eff }}$ and bolometric corrections using Flower (1996), 


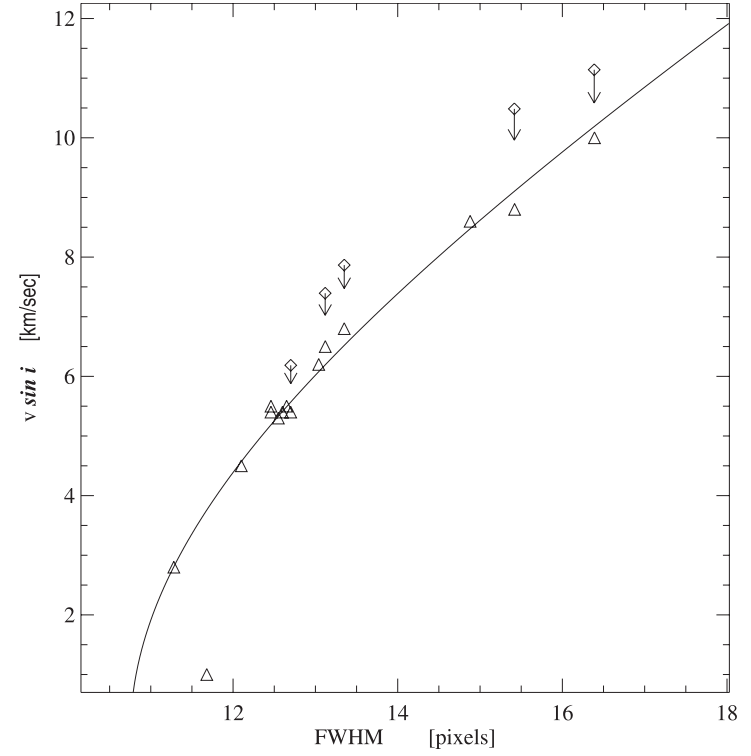

Fig. 7. $v \sin i$ vs. FWHM diagram for Hyades stars, and the fit to the data points: $v \sin i=0.82 \cdot\left(F W H M^{2}-10.75^{2}\right)^{1 / 2}$. Also equatorial velocities from periods of rotation are plotted. The latter are indicated as square symbols with the downward arrow (they are in fact upper limits for $v \sin i$ ), while $v \sin i$ data points from Paulson et al. (2003) are indicated as triangles.

Table 3. Rotational periods of Hyades from Radick et al. (1987) and rotational velocities from them inferred. The data are collected to compare equatorial with projected rotational velocities.

\begin{tabular}{ccc}
\hline \hline $\begin{array}{c}\text { van Bueren } \\
\text { id. }\end{array}$ & $\begin{array}{c}\text { Period } \\
\text { [days] }\end{array}$ & $\begin{array}{c}\text { Inferred } v \\
{\left[\mathrm{~km} \mathrm{~s}^{-1}\right]}\end{array}$ \\
\hline 31 & 5.45 & 11.1 \\
52 & 8.05 & 7.4 \\
65 & 5.87 & 10.5 \\
73 & 7.41 & 7.9 \\
97 & 8.55 & 6.2 \\
\hline
\end{tabular}

we then used Hipparcos parallaxes (ESA 1997) to transform the apparent magnitudes into luminosities.

We overplotted the rotational velocities of Table 3 on the diagram in Fig. 7. They are equatorial velocities, i.e. upper limits of the projected rotational velocity $v \sin i$, which is the quantity really related to the $F W H M$ of the cross correlation profile. On the other hand, the fact that rotational modulation is observed indicates that the inclination angle of stars cannot be too small. We therefore expect rotational velocities from period determinations to be right where they are: slightly above the projected rotational velocities.

\section{Discussion}

We showed in the previous sections that we are able to derive chromospheric fluxes and rotational velocities with great accuracy for our cluster stars. We first investigate the spread in the level of chromospheric activity in each cluster, which will tell us to which extent a single value can be used for each cluster. By comparing the pair of twin points we can then evaluate if chromospheric activity is a function of age for solar stars. Only
Table 4. Peak to peak spread of the $F_{K}^{\prime}$ fluxes within each cluster, expressed in percentage of the related mean value. The spread of the solar data is the variation during the solar cycle 21 .

\begin{tabular}{ccc}
\hline \hline Cluster & Spread & Stars \\
& & \\
\hline PRAESEPE & $59.3 \%$ & 6 \\
HYADES & $68.3 \%$ & 15 \\
IC 4651 & $60.2 \%$ & 5 \\
NGC 3680 & $31.2 \%$ & 2 \\
M 67 & $62.4 \%$ & 7 \\
\hline SUN & $43.7 \%$ & \\
\hline
\end{tabular}

after that will we be able to derive an age-activity relationship. Finally, the same is applied to rotational velocities.

\subsection{Variability within each cluster}

According to the detailed study of White \& Livingston (1981), the level of solar chromospheric activity varies of $\sim \pm 10 \%$ (from peak to peak) during the 11-year solar cycle. If we consider the data corrected for photospheric contribution, then the variation becomes of $\sim \pm 20 \%$. Analog cycles take also place in solar-type stars (see e.g., Hempelmann et al. 1996; Radick et al. 1998; Baliunas et al. 1998). This demands some caution in deriving chromospheric ages from a single spectrum, and causes scatter around the mean chromospheric-activity levels observed in each cluster, i.e. at a given age.

Giampapa et al. (2000) have shown that indeed in the old M 67 considerable variations are observed if a sample large enough is acquired: they find that approximately $30 \%$ of the solar-type stars in M 67 exhibit levels of activity that are outside the envelope given by the solar-activity cycle.

As shown in Table 4, all clusters but NGC 3680 (in which only two stars are observed) have a peak to peak spread slightly exceeding $\pm 30 \%$ of the mean chromospheric-activity level. For young clusters such as the Hyades and Praesepe we expect that part of this variation is due to the superposition of long and short-term variability, the last induced by rotation. Another huge cause of chromospheric variability in young stars is flaring activity. For M 67, however, for which we expect the members to behave much like the Sun, the scatter is 1.4 times larger than the variations during a solar cycle. This means that an additional spread of $\pm 24 \%$ has to be explained. For M 67 , whose mean $B-V$ colour is 0.62 , the measurement maximum errors are of about the $\pm 18 \%$. This may indicate that cycles similar to the 11-year solar cycle do not fully account for the dispersion of the data, with interesting implications for the study of the Earth-Sun system. Admittedly, we have observed each star only once (three consecutive times for the fainter stars); rather, we assume that the stars in our sample have been observed at only one phase of their long-term activity, which is likely different from star to star. This implies, for instance, that possible star to star differences may cause additional noise in our results. We think, however, that our procedure of overlapping the photospheric profiles eliminates most of the observational uncertainties. Before inferring firm conclusions, more data have to be collected. 

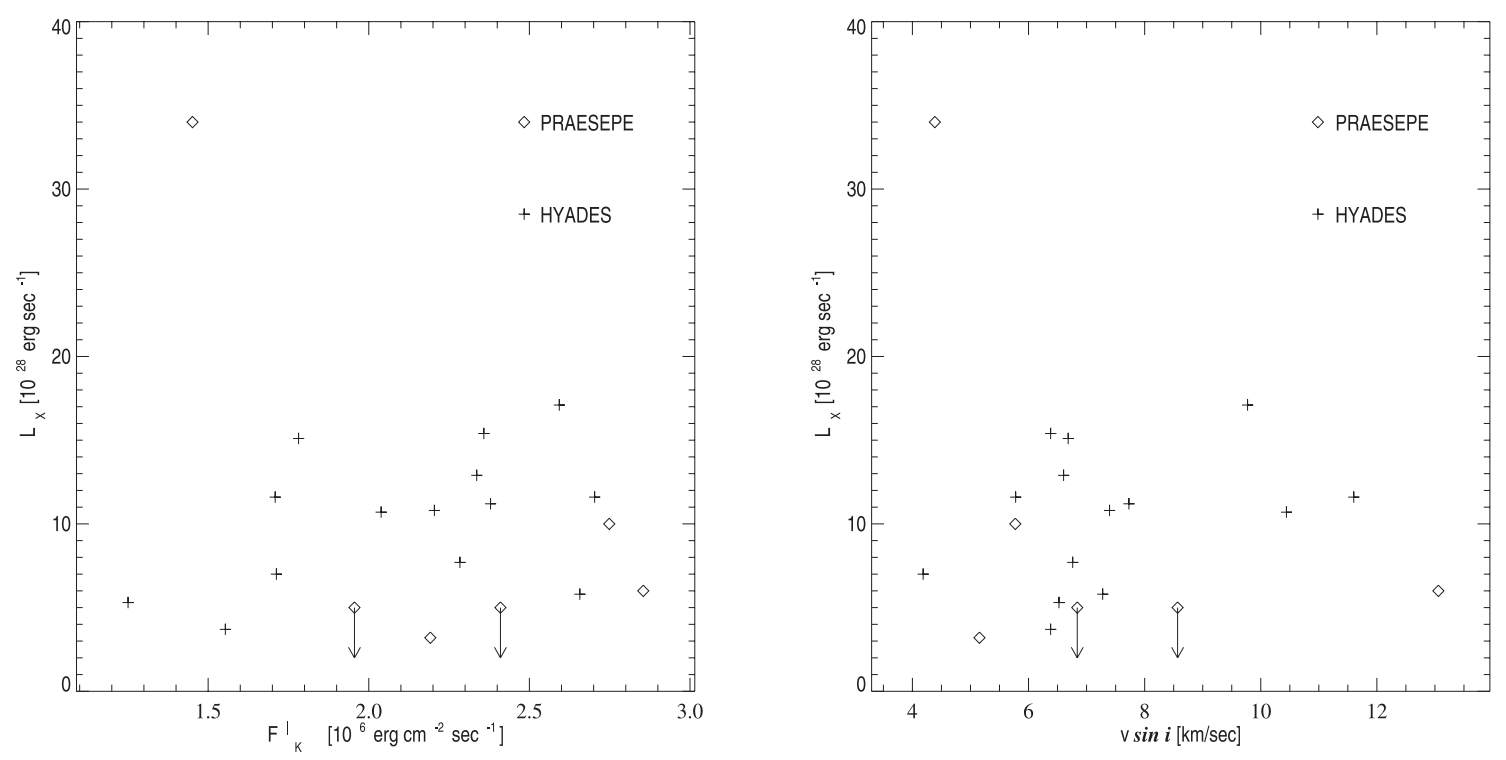

Fig. 8. X-ray luminosities vs. chromospheric activity and rotation diagrams. In neither of those can a trend be clearly seen.

Table 5. X-ray flux measurements of Praesepe and Hyades stars. All of them but one refer to the ROSAT detection in the band 0.1-2.0 keV. KW 326 was not detected by ROSAT. Its measurement, which refers to the same band, has been performed with XMM-Newton satellite.

\begin{tabular}{cc}
\hline \hline STAR & $\begin{array}{c}L_{\mathrm{X}} \\
{\left[10^{28} \mathrm{erg} / \mathrm{s}\right]}\end{array}$ \\
\hline \hline \multicolumn{2}{c}{ PRAESEPE } \\
\hline KW 49 & non det. \\
KW 100 & 6 \\
KW 326 & 3.20 \\
KW 368 & 34 \\
KW 392 & 10 \\
KW 418 & non det. \\
\hline \multicolumn{2}{c}{ HYADES } \\
\hline van Bueren 1 & 3.7 \\
van Bueren 2 & 15.1 \\
van Bueren 10 & 5.8 \\
van Bueren 15 & 12.9 \\
van Bueren 17 & 11.6 \\
van Bueren 18 & 15.4 \\
van Bueren 31 & 11.6 \\
van Bueren 49 & 7.0 \\
van Bueren 52 & 10.8 \\
van Bueren 65 & 10.7 \\
van Bueren 66 & 17.1 \\
van Bueren 73 & 11.2 \\
van Bueren 88 & - \\
van Bueren 97 & 7.7 \\
van Bueren 118 & 5.3 \\
\hline
\end{tabular}

\subsection{Hyades vs. Praesepe, IC 4651 vs. NGC 3680 , M 67 vs. Sun}

The Hyades and Praesepe clusters are particularly relevant because they have been studied also in the X-ray (Randich \& Schmitt 1995). It is at least reasonable to assume that the X-ray emission (i.e. coronal activity) and the chromospheric activity are not completely independent, since they are both related to rotation and magnetic field (see e.g. Bruevich et al. 2001, and references therein), but in Randich \& Schmitt (1995) the two coeval clusters were found to have substantially different levels of X-ray emission (Randich \& Schmitt 1995). This early result has been recently revised by Franciosini et al. (2003), who find that Praesepe stars have the same level of X-ray activity as Hyades if the central field is considered.

Our data confirm that Praesepe and Hyades stars span the same ranges of chromospheric-activity levels and $v \sin i$ values, even though our Praesepe stars have a mean X-ray luminosity significantly lower than the Hyades ones (less than a half).

In Fig. 8 the $L_{\mathrm{X}}$ vs. $v \sin i$ (right panel) and the $L_{\mathrm{X}}$ vs. $F_{K}^{\prime}$ diagram (left panel) are shown for both clusters. $L_{X}$ measurements are taken from from Stern et al. (1995) for the Hyades, and Randich \& Schmitt (1995) for Praesepe members, but KW 326, which is given in Franciosini et al. (2003)

In both panels the spread is high and no real relationship is observed. In spite of the aforementioned possible discrepancy between X-ray emission and chromospheric activity, a significant correlation likely exists. The fact that it is not seen in Fig. 8 could be due to the activity variability, since the optical and X-ray observations are not simultaneous.

When focusing on the other coeval clusters we notice in Table 1 that the activity and rotation values for IC 4651 and NGC 3680 are, as in the in the case of Praesepe and Hyades, indistinguishable. Only two stars were observed in NGC 3680, therefore its average chromospheric-activity and rotation levels cannot be considered accurate. Nevertheless it is very significant that both NGC 3680 stars have values similar to those of IC 4651. This result is particularly important because, as we shall see in the following, these clusters show a novel behaviour with respect of what thought so far.

Finally, given the excellent agreement between the mean M 67 activity values and the Sun, we can conclude that all our coeval points show very consistent average values, therefore the level of chromospheric activity is an age-driven parameter. 
The $v \sin i$ for M 67 is slightly larger than the solar one ( $\sim 3 \mathrm{~km} \mathrm{~s}^{-1}$, see Sect. 3.2); we do not consider this at the moment as a significant discrepancy, but it would be interesting to verify that M 67 cluster stars rotates indeed slightly faster than the Sun.

\subsection{The age-activity-rotation relationship.}

When the stars of about $1 M_{\odot}$ reach the ZAMS, they show a high dispersion in rotational velocity, and therefore in chromospheric-activity levels, as a result of their different Pre Main Sequence histories (see e.g. Bouvier 1997). But at $600 \mathrm{Myr}$, i.e. the youngest age we are dealing with in the present study, the magnetic braking mechanism already made the rotational velocity uniform. So, for each of our clusters, rotational velocity and chromospheric activity are expected to be all of the same order of magnitude (see Sect. 4.1). The evolution of these quantities, as it results from our data, is shown in the two panels of Fig. 9. It is very important to bear in mind that in this figure the bars are not error bars, but they represent the peak to peak spread within the clusters, i.e., the difference between the maximum and the minimum cluster values, and the points are the mean values.

As expected, chromospheric activity and rotation show the same decaying trend. Surprisingly, however, the intermediate age clusters IC 4651 and NGC 3680 have the same level of activity and rotation as the older M 67 and the Sun. We recall that these two data points are based on measurements of 7 stars, two in NGC 3680 and 5 in IC 4651, none of which shows a chromospheric activity above the most active star in M 67, i.e., the activity distribution of the 7 intermediate age stars and that of the $7 \mathrm{M} 67$ stars are virtually equal.

This means that an abrupt decay of chromospheric activity occurs between Hyades' age and $1.5 \mathrm{Gyr}$, after which a very slow decline follows, if any. Three power law decay laws are plotted over the data points of Fig. 9: the two more gentle slopes represent the $t^{-1 / 2}$ and $t^{-2 / 3}$ laws proposed respectively by Skumanich (1972) and Soderblom et al. (1991), scaled to best match our data points.

The intermediate age clusters are clearly not consistent with any of the laws so far suggested; they have an activity level lower more than a factor 2 . If the exponent of the power law is left as a free parameter, we find that the points are best fitted by the third curve plotted on the $F_{K}^{\prime}$ vs. age diagram, namely $F_{K}=0.5 \cdot t^{-2.5}+0.5$, where $F_{K}^{\prime}$ is in unit of $10^{6} \mathrm{erg} \mathrm{cm}^{-2} \mathrm{~s}^{-1}$, and the age is expressed in Gyr. Note that neither a different zero point, nor different assumptions on the age of the clusters would change our conclusion, since activity keeps constant for a time interval, the one between the intermediate age clusters and M 67, that under no acceptable assumptions can be shorter than 2 Gyr. Similar results are also claimed by Rodrigues et al. (2003).

Figure 9 recalls what is observed among field stars: the socalled Vaughan-Preston gap, i.e. the lack of nearby stars with a level of chromospheric activity intermediate between Hyades' and the Sun (Vaughan \& Preston 1980). With our results this gap has a quite natural explanation: the decline in activity is so
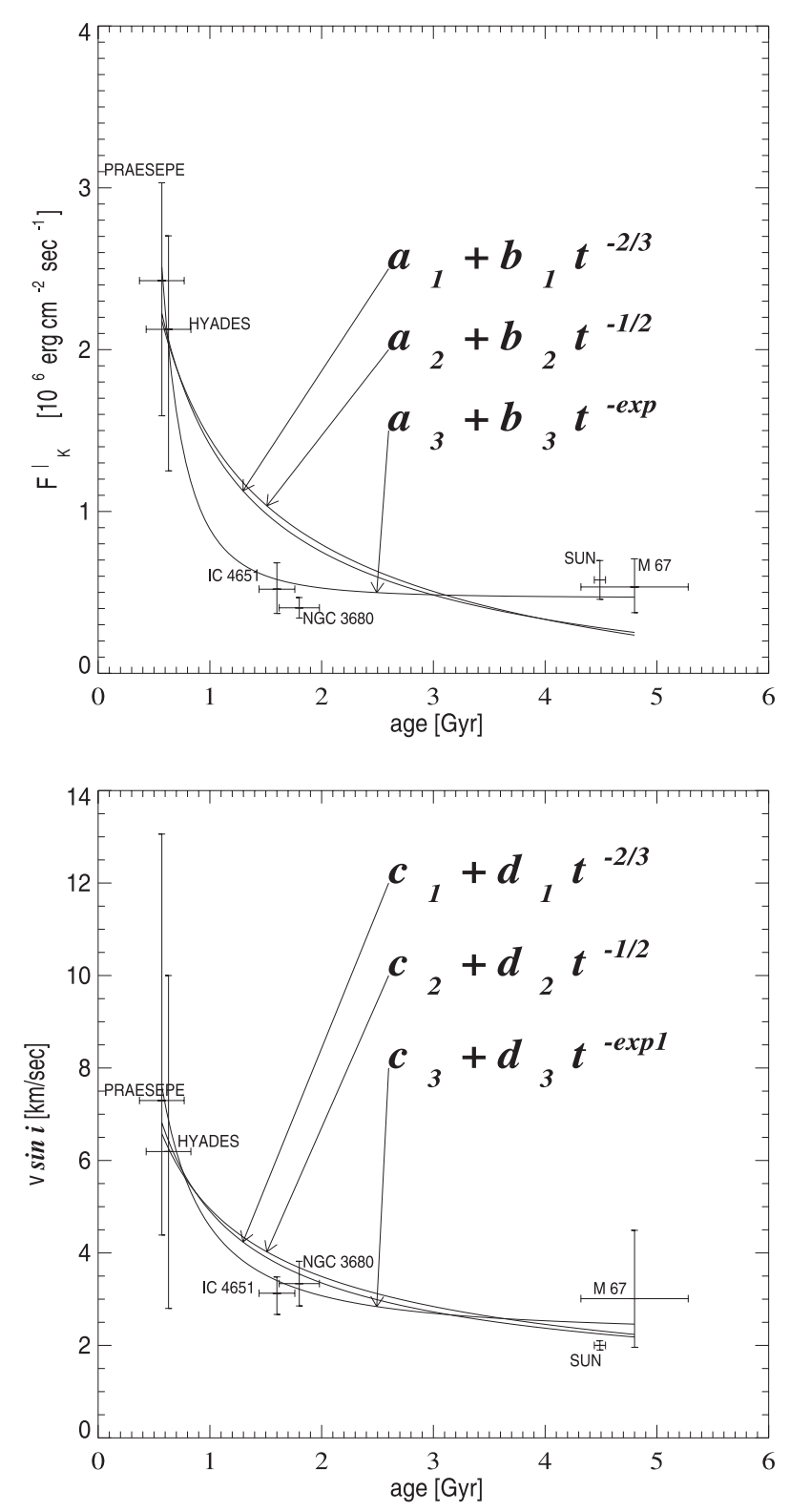

Fig. 9. The evolution of chromospheric activity (measured by $F_{K}^{\prime}$ corrected for IS absorption, upper panel) and rotation (lower panel) with age. Here the vertical bars represent the whole spread of the ordinate data within the cluster. The points are the mean values. For both quantities the surprising result is that the intermediate age clusters show the same level of chromospheric activity and mean rotational velocity of M 67 and the Sun. Since, as remarked, the bars are not error bars but peak to peak spreads, their position attests that the activity distribution of the 7 intermediate age stars and that of the 7 M 67 stars are virtually equal. For both diagrams we tried to fit three power laws, as indicated in the figure. $-2 / 3$ and $-1 / 2$ are the exponent values suggested respectively by Barry et al. (1987) and Skumanich (1972). As can be seen, they do not fit our data. By minimizing the $\chi^{2}$ we find, for the free parameters: $a_{1}=-0.38, b_{1}=1.79, a_{2}=-0.79, b_{2}=2.24, a_{3}=$ $0.47, b_{3}=0.43, \exp =2.79, c_{1}=0.71, d_{1}=4.20, c_{2}=-0.05, d_{2}=$ $5.00, c_{3}=2.23, d_{3}=2.35, \exp 1=1.47$.

abrupt to be almost a discontinuity, and only stars with ages in the range between $0.6-1.5$ Gyr may fall in it. 
As mentioned above, the previous most systematic attempt to study chromospheric activity in stars belonging to open clusters has been made by Barry et al. (1987), who obtained results quite different from ours. Some major discrepancy may exist in the measurement of the activity index. For instance, in Barry et al. (1987), the ratio between the chromospheric fluxes in the Hyades and in M 67 stars or in the Sun, is only $\sim 2.2$, while for us is larger than 4 . We find a similar ratio $(\simeq 2.4)$ if our index includes the photospheric contribution which represents a fraction of the $1-\AA$ index much larger for M 67 than for Hyades stars. In Fig. 9 the difference between ours and Barry et al.'s data is apparent. Given the large scatter of fluxes observed in each cluster, we cannot, however, exclude that the discrepancy is due to differences in the sample observed. We tend to consider this possibility unlikely, considering the large number of stars observed. Barry et al. have observed 13 stars in the Hyades and 16 in M 67 and we have observed 15 stars in Hyades and 7 in M 67. These authors measure also a chromospheric-activity index for the intermediate age cluster NGC 752 only 1.4 times lower than the Hyades and 1.5 times bigger than M 67; this would imply a level of chromospheric activity in the middle between the Hyades and the old stars plateau, right in the Vaughan-Preston gap. Carraro \& Chiosi (1994), so far used as age reference, evaluate the age of NGC 752 comparable, but slightly younger than IC 4651 (1.5 vs. $1.6 \mathrm{Gyr}$ ).

Before going into speculations about the difference between our and Barry et al.'s results, the NGC 752 chromospheric-activity level should be proven by means of high-resolution spectroscopy in solar analogs.

\subsection{The impact of our results on the determination of ages and on the age of galactic population}

According to the picture we have drawn in the last section, stars can be divided in three groups on the basis of their chromospheric-activity levels:

- the youngest stars, with $F_{K}^{\prime} \gtrsim 1.2 \times 10^{6} \mathrm{erg} \mathrm{cm}^{-2} \mathrm{~s}^{-1}$

- the stars with $F_{K}^{\prime}$ between $\sim 0.7$ and $\sim 1.2 \mathrm{erg} \mathrm{cm}^{-2} \mathrm{~s}^{-1}$

- the oldest stars, with $F_{K}^{\prime} \lesssim 0.7 \times 10^{6} \mathrm{erg} \mathrm{cm}^{-2} \mathrm{~s}^{-1}$.

Among our target stars, those in Praesepe and Hyades belong the first group, all the others to the third one. Let us call the upper age limit of the first ensemble $t_{1}$ and the lower age limit of second $t_{2}$. We don't know the exact values of $t_{1}$ and $t_{2}$. We only know that $0.6 \mathrm{Gyr}<t_{1}<t_{2}<1.6 \mathrm{Gyr}$.

In Fig. 10 we plot the histogram for 44 solar-type stars (spectral type between G0 and G5) in the solar neighborhood taken from Pasquini (1992). The numbers of stars belonging to each group are respectively: 8,4 and 34 . The result confirms what expected: most of the stars have an age older than $t_{2}$, much less are younger than $t_{1}$, less than $10 \%$ have ages between $t_{1}$ and $t_{2}$, i.e. those in the Vaughan-Preston gap. In addition, the standard deviation of the $F_{K}^{\prime}$ values of the 34 less active stars in the sample of Pasquini (1992) is very similar to that of the 14 targets belonging to the three intermediate age and old clusters IC 4651, NGC 3680 and M 67: $0.12 \times 10^{6} \mathrm{erg} \mathrm{cm}^{-2} \mathrm{~s}^{-1}$. Unfortunately our results indicate that an accurate age determination based on chromospheric activity may not be obtained

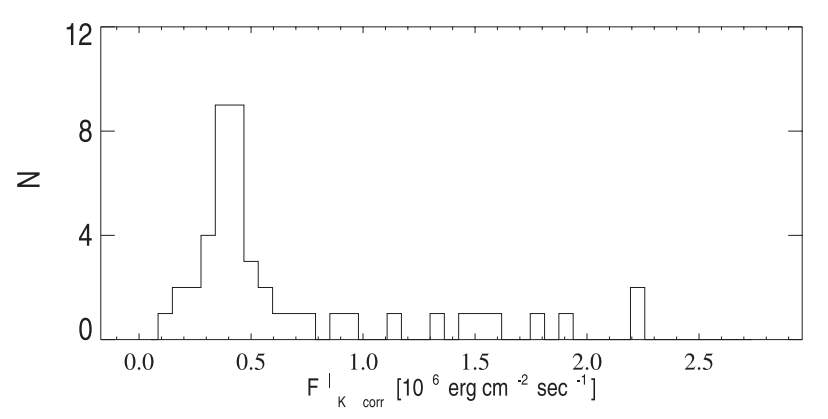

Fig. 10. Histogram of the chromospheric-activity levels in the sample of stars from Pasquini (1992).

for stars older than $\sim 1.5$ Gyr. Once $t_{1}$ and $t_{2}$ are determined precisely, much could be said about the recent Star Formation History in the solar neighborhood, provided that initial conditions do not dominate the fast decline of chromospheric activity occurring between $t_{1}$ and $t_{2}$.

\section{Conclusions}

We have studied the evolution of chromospheric activity and rotation in solar-type stars by analysing VLT and KECK highresolution spectra of 35 stars belonging to 5 open clusters and published solar data. As a measure of chromospheric activity we have used $F_{K}^{\prime}$, i.e. the equivalent width of the $\mathrm{Ca}$ II $\mathrm{K}$ line emission core corrected for photospheric contribution and transformed into flux at the stellar surface. Our data set can be divided in three age bins: 6 Praesepe stars and 15 Hyades stars at $0.6 \mathrm{Gyr} ; 5$ IC 4651 stars and 2 stars in NGC 3680, at about $1.7 \mathrm{Gyr}$; and finally $7 \mathrm{M} 67$ stars and the Sun, at about 4.5 Gyr. We undertook the present research mainly to ascertain a decay law which allows measuring chromospheric ages in field stars. We have found that stars within each age bin show indeed the same mean level of chromospheric activity, confirming that it is possible to search for a chromospheric activity-age relationship. Stars in the youngest bin have chromospheric fluxes ranging between 1.2 and $2.8 \times 10^{6} \mathrm{erg} \mathrm{cm}^{-2} \mathrm{~s}^{-1}$. All the other stars have values ranging between 0.3 and $0.7 \times 10^{6} \mathrm{erg} \mathrm{cm}^{-2} \mathrm{~s}^{-1}$. The most important result is that there is no difference in the distribution of chromospheric-activity levels between the intermediate and the oldest age bin, even though difference of ages of the two bins is as large as $\sim 3$ Gyr. We obtain a very similar conclusion about the angular momentum evolution, with the only difference that the gap between the youngest bin and the others is less pronounced. A fast decay of chromospheric activity occurs roughly between 0.6 and 1.5 Gyr of Main Sequence lifetime, after which a kind of plateau appears.

No power law so far proposed can fit our data. The best match curve we computed, namely $F_{K}^{\prime}=0.5 \cdot t^{-2.4}+0.4$, where $F_{K}^{\prime}$ is in unit of $10^{6} \mathrm{erg} \mathrm{cm}^{-2} \mathrm{~s}^{-1}$, and the age is expressed in Gyr, can reproduce this trend.

Coupling this trend with the observational fact that in each of the clusters observed the star to star activity level vary almost by a factor 2 , our results indicate that the determination of chromospheric ages for field stars older than $\sim 1.5 \mathrm{Gyr}$ is extremely challenging, or even potentially misleading, 
in particular if only one or a few activity measurements are available.

Acknowledgements. We are greatly indebted to Bill Cochran, Artie Hatzes and Diane Paulson, for making available the HIRES spectra of Hyades' stars, to Piercarlo Bonifacio, for computing the synthetic CaII solar spectrum, and to Claudio Melo, for sharing the code and the digital mask for the computation of the cross correlation profiles. This paper took advantage of the useful comments of Diane Paulson and of the impressive work of the referee, Dr. Messina. We also thank Nick Pettefar for the careful reading of the manuscript.

\section{References}

Allen, C. W. 1973, Astrophysical Quantities (Athlone Press)

Anthony Twarog, B., \& Twarog, B. A. 1987, AJ, 94, 1222

Anthony Twarog, B., Mukherjee, K., Caldwell, N., \& Twarog, B. A. 1988, AJ, 95, 1453

Ballester, P., Modigliani, A., Boitquin, O., et al. 2000, ESO Messenger, 101, 31

Baliunas, S. L., Donahue, R. A., Soon, W., \& Henry, G. H. 1998, ASP Conf. Ser., 154, 153

Barrado y Navascues, D., Stauffer, J. R., \& Randich, S. 1997, MmSAI, 68, 985

Barry, D. C., Cromwell, R. H., \& Hege, E. K. 1987, ApJ, 315, 264

Benz, W., \& Mayor, M. 1981, A\&A, 93, 235

Blanco, C., Catalano, S., Marilli, E., \& Rodonó, M. 1974, A\&A, 33, 257

Bouvier, J. 1997, Mem.S.A.It., 68, 881

Bruevich, A. E., Katsova, M. M., \& Sokolov, D. D. 2001, Astron. Rep., 45, 718

Carraro, G., \& Chiosi, C. 1994, A\&A, 287, 761

Carraro, G., Girardi, L., Bressan, A., \& Chiosi, C. 1996, A\&A, 305, 849

Catalano, S. 1978, A\&A, 80, 317

Cochran, W. D., Hatzes, A. P., Paulson, D. B. 2002, AJ, 124, 565

Dekker, H., D’Odorico, S., Kaufer, A., Delabre, B., \& Kotzlowski, H. 2000, SPIE, 4008, 534

Duncan, D. K., Baliunas, S. L., Noyes, R. W., et al. 1984, PASP, 96, 707

Dupree, A. K., Whitney, B. A., \& Pasquini, L. 1999, ApJ, 520, 751

Eggen, O. J. 1969, ApJ, 155, 439

Eggen, O. J. 1971, ApJ, 166, 87

ESA 1997, The Hipparcos and Tycho Catalogues, ESA SP-1200

Flower, P. J. 1996, ApJ, 469, 355

Franciosini, E., Randich, S., \& Pallavicini, R. 2003, A\&A, 405, 551

Giampapa, M. S., Radick, R. R., Hall, J. C., \& Baliunas, S. L. 2000, American Astronomical Society, SPD meeting 32, 02/12/2000

Gray, D. F. 1992, The observation and analysis of stellar photospheres (Cambridge Astrophphys. Ser.)

Guenther, D. B. 1989, ApJ, 339, 1156

Hempelmann, A., Scmhmitt, J. H. M. M., \& Stepien, K. 1996, A\&A, 305,284

Henry, T. J., Soderblom, D. R., Donahue, R. A., \& Baliunas, S. L. 1996, AJ, 11, 439

Jones, B. F., \& Cudworth, K. 1983, AJ, 88, 215

Jones, B. F., \& Stauffer, J. R. 1991, AJ, 102, 1080
Klein Wassink, W. J. 1927, Publ. Kapteyn Astron. Lab. Groningen, 41,1

La Bonte, B. J. 1986, ApJS, 62, 229

Latham, D. W., Mathieu, R. D., Milone, A. A. E., \& Davis, R. J. 1992, IAUS, 151, 471

Linsky, J. L., \& Avrett, E. H. 1970, PASP, 82, 169

Linsky, J. L., \& Ayres, T. R. 1978, ApJ, 220, 619

Linsky, J. L., McClintock, W., Robertson, R. M., \& Worden, S. P. 1979, ApJS, 41, 47

Meibom, S., Andersen, J., \& Nordström, B. 2002, A\&A, 386, 187

Melo, C., Pasquini, L., \& De Medeiros, J. R. 2001, A\&A, 375, 851

Mermilliod, J. C. 1981, A\&A, 97, 235

Montgomery, K. A., Marschall, L. A., \& Janes, K. A. 1993, AJ, 106, 181

Nordström, B., Andersen, J., \& Andersen, M. I. 1996, A\&AS, 118, 407

Nordström, B., Andersen, J., \& Andersen, M. I. 1997, A\&A, 322, 460

Noyes, R. W., Hartmann, S., Baliunas, S. L., Duncan, D. K., \& Vaughan, A. H. 1984, ApJ, 279, 763

Parker, E. N. 1970, ARA\&A, 8, 1

Pasquini, L. 1985, Ph.D. Thesis

Pasquini, L. 1992, A\&A, 266, 347

Pasquini, L., Pallavicini, R., \& Pakull, M. 1988, A\&A, 191, 253

Pasquini, L., Brocato, E., \& Pallavicini, R. 1990, A\&A, 234, 277

Pasquini, L., Randich, S., Zoccali, M., et al. 2003, Detailed Chemical composistion of the open Cluster IC 4651: Metals, $\alpha$ elements and $\mathrm{Li}$, in preparation

Paulson, D. B., Saar, S. H., Cochran, W. D., \& Hatzes, A. 2002, ApJ, 124,572

Paulson, D. B., Snenden, C., \& Cochran, W. D. 2003, AJ, 125, 3185

Perryman, M. A. C., Brown, A. G. A., Lebreton, Y., et al. 1998, A\&A, 331,81

Radick, R. R., Thompson, D. T., Lockwood, G. W., Duncan, D. K., \& Baggett, W. E. 1987, ApJ, 321, 459

Radick, R. R., Lockwood, G. W., Stiff, B. A., \& Baliunas, S. L. 1998, ApJS, 118, 239

Randich, S., \& Scmhmitt, J. H. M. M. 1995, A\&A, 298, 115

Rocha Pinto, H. J., Scalo, J., Maciel, W. J., \& Flynn, C. 2000, A\&A, 358, 850 (Paper I)

Rocha Pinto, H. J., Scalo, J., Maciel, W. J., \& Flynn, C. 2000, A\&A, 358, 869 (Paper II)

Rodrigues, G. R. K., Lyra, W., \& Porto de Mello, G. F. 2003, Boletim da Sociedade Astronomica Brasileira, 23, 131

Sanders, W. L. 1977, A\&AS, 27, 89

Skumanich, A. 1972, ApJ, 171, 565

Soderblom, D. R., Duncan, D. K., \& Johnson, D. R. H. 1991, ApJ, 375,722

Stern, R. A., Schmitt, J. H. M. M., \& Kahabka, P. T. 1995, ApJ, 448, 683

Straizis, V., \& Valiuga, G. 1994, BaltA, 3, 282

Strassmeier, K. G., Washuettl, A., Granzer, T., Scheck, M., \& Weber, M. 2000, A\&AS, 142, 275

Taylor, B. J., \& Joner, M. D. 2002, AAS, 200, 902

van Bueren, H. G. 1952, Bull. Astron. Inst. Netherlands, 11, 385

van den Bergh, S., McClure, R.D. 1980, A\&A 88, 360

Vaughan, A. H., \& Preston, G. W. 1980, PASP, 92, 385

White, O. R., \& Livingston, W. C. 1981, ApJ, 249, 798

Wilson, O. C. 1963, ApJ, 138, 832 